\title{
Derivation of a quantitative measure of insulin sensitivity from the intravenous tolbutamide test using the minimal model of glucose dynamics
}

\author{
N. M.Shennan, I. F. Godsland and V. Wynn \\ The Cavendish Clinic, London, UK
}

\begin{abstract}
Summary. Using the decay phase of the glucose response during an intravenous tolbutamide test, a minimal model of glucose dynamics was used to calculate a value for an "index of insulin sensitivity". This index describes the efficiency of insulin in accelerating the instantaneous rate of glucose disposal, and provides a measure of insulin resistance. The validity of estimates of the index of insulin sensitivity obtained from the intravenous tolbutamide test have been assessed with reference to estimates of this index derived from the intravenous glucose tolerance test for which the model was originally designed. There were three studies: (A) estimates of the index of insulin sensitivity obtained from the intravenous tolbutamide test in a group of normal, healthy men and women were compared with results obtained in a comparable group of subjects using the intravenous glucose tolerance test. The two methods gave estimates of the index of insulin sensitivity that were identical; (B) A group of patients taking methandienone, an anabolic steroid previously shown to cause marked insulin resistance, were tested whilst taking the steroid and either before, or at least two months after treatment. Each patient was tested by both intravenous tolbutamide test and intravenous glucose tolerance test on both occasions. Estimates of the index of insulin sensitivity from intravenous glucose tolerance or intravenous tolbutamide procedures both on and off treatment were significantly correlated (off treatment: $r_{s}=0.71, n=9, p<0.05$; on treat-
\end{abstract}

ment: $\left.r_{s}=0.69, n=9, p<0.05\right)$; (C) A group of patients undergoing investigations for suspected disturbances in carbohydrate metabolism was studied, each patient having had both an intravenous tolbutamide and intravenous glucose tolerance test. The group studied included patients in whom a degree of insulin resistance would be expected. Estimates of the index of insulin sensitivity from the two methods were closely correlated $\left(r_{s}=0.95, n=25, p<0.001\right)$. This strong, identical correlation obtained between the intravenous glucose tolerance and intravenous tolbutamide tolerance estimates of index of insulin sensitivity in studies $B$ and $C$ over a wide range of values [intravenous tolbutamide tolerance test: 0.11-1.07 $\mathrm{min}^{-1} \mathrm{U}^{-1} 1$; intravenous glucose tolerance test: $\left.0.12-1.06 \mathrm{~min}^{-1} \mathrm{U}^{-1} \mathrm{l}\right]$. This suggests that the intravenous tolbutamide estimates of index of insulin sensitivity are closely comparable to those derived from intravenous glucose tolerance test over a broad range of insulin sensitivities. We suggest that the use of intravenous tolbutamide to induce a dynamic change in insulin-glucose relationships, and mathematical modelling of those dynamics, can provide a valuable, quantitative measure of insulin sensitivity in a variety of clinical situations.

Key words: Insulin sensitivity, intravenous tolbutamide test, intravenous glucose tolerance test, insulin action, glucose dynamics, mathematical modelling.
The minimal model of glucose dynamics has been used to derive estimates of an index of insulin sensitivity $\left(S_{I}\right)$ from the intravenous glucose tolerance test (IVGTT) in man [1]. $S_{I}$ is defined mathematically as a ratio of two rate constants governing (a) the action of insulin via the plasma from a remote compartment, $I$, on the sites of active glucose disposal and (b) the egress of insulin from this remote compartment (and hence the diminution of its action). Such a quantity is potentially useful to clinicians and research workers, as it offers a quantitative measure of the effectiveness of insulin in accelerating glucose disposal.
Although the minimal model was originally designed for use where plasma glucose concentrations are elevated, it is theoretically used whenever plasma glucose concentrations are falling because of a rise in insulin secretion. During an intravenous tolbutamide test (IVTT), where sulphonylurea stimulation of the islet B cell increases insulin secretion, the plasma glucose concentration falls from the basal level to a nadir. During a fall in plasma glucose concentrations following such an increase in insulin secretion, it has been demonstrated [2-6] that the plasma concentrations of the principal counter-regulatory hormones (glucagon, 
cortisol, catecholamines and growth hormone), barely respond for approximately $30 \mathrm{~min}$, at which point the plasma glucose is still roughly $20 \%$ above its eventual nadir. Furthermore, it has been demonstrated that glucose production drops continuously over this period [2] consistent with the inhibitory effect of elevated plasma insulin concentrations. Thus, with glucose disposal unopposed, a continuous rise in net glucose utilisation is observed in response to a sustained elevation in plasma insulin concentrations. It would appear, therefore, that the action of insulin on net glucose disposal within the first $30 \mathrm{~min}$ following the administration of tolbutamide is unconfounded by the effects of other counter-regulatory glucostatic hormones. Consequently, application of the minimal model during this period to derive estimates of $S_{I}$ is valid.

In the present study, we have compared estimates of $S_{I}$ derived from the modelling of plasma glucose concentrations obtained during both IVTTs and IVGTTs in three groups of subjects in which a wide range of insulin sensitivities may be expected. We have excluded cases of obesity or diabetes mellitus from the present study. However, we are currently studying these other conditions using the techniques described.

\section{Subjects and methods}

\section{Subjects}

The three groups of subjects studied were as follows:

Study $A$. Normal healthy non-obese men and women were selected following a thorough medical examination, including reference to exhaustive lists of drugs and diagnoses associated with disturbances of carbohydrate metabolism. Fifty-eight cases were studied, $30 \mathrm{hav}$ ing IVGTTs (15 male, 15 female) and 28 having IVTTs (13 male, 15 female). There were no significant differences in age and body mass index (BMI) between the IVGTT study group (age range: 19-58 years; BMI range: $19.4-23.0 \mathrm{~kg} / \mathrm{m}^{-2}$ ) and IVTT study group (age range: $20-51$ years; BMI range: $18.5-24.3 \mathrm{~kg} / \mathrm{m}^{-2}$ ).

Study $B$. Nine patients ( 2 men, 7 women) were studied whilst on treatment and either before or after treatment with the anabolic steroid, methandienone. The indication for this treatment was severe weight loss following a period of negative nitrogen balance associated with post-operative recovery or anorexia. The patients had both an IVGTT and an IVTT. In 7 cases tests were carried out prior to commencing treatment and then on treatment. The remainder were tested on treatment, and then when off treatment for at least 2 months. Dosages of methandienone were $10 \mathrm{mg}$ (4 subjects), $15 \mathrm{mg}$ ( 1 subject) or $20 \mathrm{mg}$ ( 4 subjects) per day. This group has been discussed in detail elsewhere with respect to the mathematical modelling of IVGTT data [8]. In the current analysis, the group has been examined in order to compare estimates of $S_{I}$ derived from both IVTT and IVGTT procedures. These experiments were carried out around 1960 , at a time when ethical committee approval had not yet been in stitutionalised in the UK.

Study C. Twenty-five patients undergoing investigation for suspected disturbances in carbohydrate metabolism were included in this study. Patients had both an IVGTT and an IVTT. Two men proved to be healthy, 5 men and 2 women had previously shown evidence of reactive hypoglycaemia, 3 men had glycosuria, 1 man was acromegalic and 2 women had Cushing's syndrome. The group also in- cluded seven women taking a variety of oral contraceptives, and 1 woman and 2 female transsexuals receiving testosterone enanthate.

All volunteers and patients had the procedures explained to them and gave their informed consent. Patients in study B were being investigated for the metabolic effects of methandienone and related compounds. The clinical decision to administer methandienone was the responsibility of one of the authors (VW). Patients in study $\mathrm{C}$ were undergoing detailed investigations into pancreatic function and glucose dissimilation in relation to diagnosis and monitoring of disorders of carbohydrate metabolism. Use of the procedures described in this study has the approval of the Paddington and North Kensington Area Health Authority Ethical Committee.

\section{Methods}

Prior to testing, all subjects had followed a diet containing approximately $300 \mathrm{~g}$ of carbohydrate per day for 3 days and had fasted from 21.00 hours the previous evening. Tests commenced at 09.00 hours and patients remained semi-recumbent throughout each test. Cannulae were introduced into an antecubital vein in both arms under local anaesthetic, and after $20 \mathrm{~min}$ bed rest two blood samples were withdrawn $10 \mathrm{~min}$ apart from one cannula for the determination of basal glucose and insulin concentrations. The mean concentrations for these samples were taken as the basal values. Throughout both procedures, heparin in saline was used to ensure patency of the sampling-site cannula. Samples were collected into tubes containing lithium heparin as an anti-coagulant, and were immediately centrifuged. The plasma was separated, and aliquots for the determination of glucose concentrations were stored at $4^{\circ} \mathrm{C}$ and assayed on the same day. Those for plasma insulin determination were stored at $-20^{\circ} \mathrm{C}$ and assayed within 1 month.

IVGTT procedure. Glucose was injected into the cannula contralateral to that from which the baseline samples were taken. Dextrose ( $50 \%$ in water) was injected at $0.5 \mathrm{~g}$ per $\mathrm{kg}$ body weight over $2 \mathrm{~min}$, the cannula position having been checked to ensure that extravasation did not occur. Samples $(10 \mathrm{ml})$ of venous blood were withdrawn from the sampling site on the contralateral arm. They were taken from the start of glucose injection: $1,2,3,5,7,10,20,30,45,60,75$, $90 \mathrm{~min}$.

IVTT procedure. An injection of tolbutamide $(1 \mathrm{~g})$ in saline was given over 1 min into the cannula contralateral to that from which the baseline samples were taken. Samples $(10 \mathrm{ml})$ of venous blood were withdrawn from the contralateral arm from the start of tolbutamide injection: $1,2,3,5,7,10,20,30,45,60,75,90,105,120 \mathrm{~min}$.

Biochemical analyses. Plasma glucose concentrations were measured on a Technicon Autoanalyser using the glucose oxidase method of Cramp [10]. Within and between assay coefficients of variation (CVs) were $1.6 \%$ and $1.6 \%$, respectively, at a mean glucose concentration of $8 \mathrm{mmol} / \mathrm{l}$. Plasma insulin levels were measured in duplicate by the specific radioimmunoassay of Albano et al. [11], with single incubation and charcoal separation, and reagent conditions selected to give maximum sensitivity over the range of elevated insulin concentrations likely to be encountered during an IVGTT or IVTT. The within and between assay CVs for this assay were $6.6 \%$ and $9.8 \%$, respectively. In addition, fasting insulin concentrations were determined by a disequilibrium modification of the insulin method, with the dilution of reagents increased to give a tenfold increase in sensitivity.

Modelling analysis. The minimal model of glucose dynamics has been described in detail elsewhere by the originators of the technique $[12,13]$, and by ourselves in a previous study of the metabolic effects of the anabolic steroid, methandienone [8].

Briefly, the minimal model of glucose dynamics employs two first-order non-linear differential equations, one describing the influence of insulin, acting from a compartment, $I$, remote from the plas$\mathrm{ma}$, on the disappearance of glucose from the plasma, and another which predicts the change in the moment-to-moment action of insu- 
lin, based on its input to the sites of glucose disposal, and on its own degradation.

In its application to the IVGTT, during which there is marked hyperglycaemia, the minimal model has to take into account both insulin-dependent and insulin-independent glucose disposal. Two parameters are thus derived which quantify these different aspects of glucose disposal, namely the insulin-dependent parameter, $S_{I}$ and the insulin-independent parameter, $S_{G}$.

To derive the differential equations that describe the phase of insulin-induced glucose concentration decay following stimulation of insulin secretion by intravenous tolbutamide, we begin with the basic model originally established to describe glucose dynamics following intravenous injection of glucose.

$\dot{G}=-(k 1+k 5) \cdot G_{t}-X_{t} \cdot G_{t}+p 4$

where:

$\dot{G}$ equals the change in plasma glucose concentration per unit time; $k 1$ equals the rate constant governing insulin-independent disposal of glucose into the peripheral tissues, $k 5$ the rate constant governing the insulin-independent net hepatic glucose balance, $G_{t}$ the plasma glucose concentration at time, $t ; X_{t}$ the net action of insulin exerted from the remote compartment on the sites of glucose disposal, and $p 4$ the extrapolated net glucose balance at zero time. Algebraically, this is equivalent to the expression $(k 1+k 5) \cdot G_{b}$, where $G_{b}$ is the basal glucose concentration.

The results of studies by Rizza et al. [14], and by Bergman and Bucolo [15] suggest that, at plasma insulin concentrations normally experienced during the phase of decline in plasma glucose concentrations in an IVTT, hepatic glucose production would be rapidly and completely suppressed. Thus, it is assumed that the term $p 4$ does not contribute significantly to the plasma glucose dynamics during this time. In addition, results from studies by Best et al. [16] can be used to calculate that, during the same period of an IVIT, $95 \%$ of the change in net glucose disposal rate is accounted for by insulindependent glucose uptake. It is thus reasonable to assume that changes in insulin-independent glucose disposal make a negligible contribution to glucose dynamics during this phase. Thus, we arrive at the following equation:

$\dot{G}=-X_{t} \cdot G_{t}$

Using the same notation of Eq. (1) above, the instantaneous change in insulin action is given by another first-order non-linear differential equation:

$\dot{X}=-k 3 \cdot X_{t}+k 2 \cdot(k 4+k 6) \cdot I_{t}$

where:

$\dot{X}$ equal change in insulin action from the remote compartment per unit time; $k 2$ the rate constant governing the ingress of insulin to the compartment, $I$, remote from the plasma; $k 3$ the rate constant describing the declining action of insulin from the remote compartment; $k 4$ the rate constant describing the remote action of insulin on peripheral glucose disposal; $k 6$ the rate constant describing the remote action of insulin on the hepatic glucose balance; $I_{i}$ the plasma insulin concentration at time, $t ; X_{t}$ the net remote insulin action at time, $t$.

Modelling of IVTT glucose concentrations on the plasma insulin concentrations may then provide solutions of Eqs. (2) and (3) in terms of values for the combined rate constants $[k 2 \cdot(k 4+k 6)]$ which govern insulin-mediated glucose disposal and $k 3$ which governs the declining action of insulin in the remote compartment.

The sensitivity of the mechanism of glucose disposal to insulin can then be expressed as a ratio of these rate constants:

$S_{I}=[k 2 \cdot(k 4+k 6)] / k 3$

$S_{I}$ will then have units of $\min ^{-1} \mathrm{U}^{-1} 1$.
Computational methods. All analyses were performed on a DEC PDP-11/23 minicomputer running on an RSX-11M operating system. Reiterative solution of the differential equations by nonlinear least-squares estimation was performed using a program written in FORTRAN-77 and PDP-11 MACRO Assembler and based on the algorithm of Marquardt [17]. Fibonacci [18], and gradient [19] searches were used to optimise the interim parameter estimates until the minimal value of the residual sums of squares was found. Revised parameter estimates between iterations were derived by expansion of the increment given by:

$\Delta \mathbf{p}=\left[\mathbf{S}^{\mathrm{T}} \mathbf{R}^{-1} \mathbf{S}\right]^{-1} \mathbf{S}^{\mathrm{T}} \mathbf{R}^{-1} \Delta \mathbf{z}$

where:

$\mathbf{S}$ is the sensitivity matrix, composed of the gradient equations in terms of each parameter to be estimated; $\mathbf{R}$ is the matrix of measurement errors; $\Delta \mathbf{z}$ is the vector of differences between observed and model-derived glucose values at each time point, for the specific parameter estimates of the $\mathbf{n}^{\text {th }}$ iteration.

The increment, $\Delta \mathbf{p}$, was then used to update the matrix of parameter estimates prior to the next $\left(\mathbf{n}^{\text {th }}\right)$ iteration by:

$\mathbf{p}^{\mathrm{n}}=\mathbf{p}^{\mathrm{n}-1}+\Delta \mathbf{p}$

The measurement error matrix was constructed assuming a constant, mean within-batch coefficient of variation of the plasma glucose assay of $1.6 \%$, with an extra, presumed additive, component due to sample-timing and possible cross-contamination of $1 \%$, giving a total measurement error variation of $2.6 \%$ at each time point, $t$. It was also assumed that errors were non-systematic and Gaussian with a mean of 0 . These criteria were also used to compute the measurement error sums of squares described below.

The program was written with a subroutine to determine the change in the residual sums of squares $\left(\mathrm{SS}_{r}\right)$ computed for the difference between the observed and the model-predicted glucose values from the Monte Carlo simulation using the final parameter estimates. This change was evaluated over every five iterations, in order to establish that point at which the SS, did not improve successively by more than $1 \%$, and cumulatively by more than $2.5 \%$. In general, this was achieved within 35 iterations per individual. Final fractional standard deviation (FSD) estimates, as indications of the accuracy of the individual parameter estimates, were computed at convergence for each reiterative analysis from the principal diagonal of the Fisher information matrix, $\mathbf{J}$, which was formed according to Di Stefano [18], and Carson et al. [20]. In addition the $\mathrm{SS}_{r}$ from the fitting of the simulated to the observed glucose data were compared to the error sums of squares $\mathrm{SS}_{m}$ (computed from the criteria above) for the plasma glucose measurements at the end of each individual analysis. Bergman et al. [21] have advocated the use of the index $\sqrt{\left[\mathrm{SS}_{r} / \mathrm{SS}_{m}\right]}$ as an indicator of the accuracy with which a given model approximates the observed data, and this has been used to compare the numerical efficiency of different models. This approach has been adopted in the current study, and the term "suitability index" has been used for brevity.

Although plasma glucose and insulin data in the IVTT were collected from 0 to $20 \mathrm{~min}$, in no individual did the plasma glucose profile show any consistent decrease in the first $3 \mathrm{~min}$ after tolbutamide administration, even though plasma insulin concentrations were already considerably higher than the basal level. This delay corresponds to that period in which insulin distribution is taking place, and only after this has occurred will the action of insulin on plasma glucose be measurable. Consequently, only data spanning the period from 3 to $20 \mathrm{~min}$ were used. To accomplish this, the algorithm incorporated zero-weighting, derived from a Kronecker delta sequence [22], such that:

$w(t)=1$ if $3 \leq t \leq 20 \mathrm{~min}$

$w(t)=0$ otherwise.

All other statistical analyses were performed using the Statistical Package for the Social Sciences, adapted for the PDP-11 [23]. 
Table 1. Medians of age (years), BMI $\left(\mathrm{kg} / \mathrm{m}^{-2}\right), S_{l}\left(\min ^{-1} \mathrm{U}^{-1} \mathrm{l}\right)$, fractional standard deviation (FSD, \%) of $S$ sums of squares of measurement errors $\left(\mathrm{SS}_{m}\right)$, and of residuals $\left(\mathrm{SS}_{r}\right)$, and the suitability index $\left(\sqrt{\left[\mathrm{SS}_{r} / \mathrm{SS}_{m}\right]}\right)$ of Study $\mathrm{A}$. The suitability index is a measure of model goodnessof-fit. Actual ranges are given throughout

\begin{tabular}{llllllllll}
\hline Sex & Test & Age & BMI & \multicolumn{1}{c}{$S_{I}$} & FSD (\%) & $n$ & SS $_{m}$ & SS & Suitability index \\
\hline Males & IVGTT & 26 & 22.5 & 0.92 & 5.3 & 15 & 3.29 & 6.52 & 1.42 \\
Range: & & $21-58$ & $20.9-23.0$ & $0.86-0.99$ & $3.3-6.0$ & & $2.23-3.85$ & $4.92-7.18$ & $1.13-1.78$ \\
Males & IVTT & 26 & 22.0 & 0.88 & 5.3 & 13 & 1.64 & 3.52 & 1.45 \\
Range: & & $22-51$ & $21.3-24.3$ & $0.82-0.99$ & $3.1-6.0$ & & $1.02-2.07$ & $1.97-4.29$ & $1.21-1.73$ \\
Females & IVGTT & 23 & 20.7 & 0.92 & 5.1 & 15 & 2.99 & 5.87 & 1.37 \\
Range: & & $19-47$ & $19.4-21.7$ & $0.89-1.09$ & $3.0-6.0$ & & $1.88-3.85$ & $4.60-6.79$ & $1.09-1.58$ \\
Females & IVTT & 24 & 20.2 & 0.90 & 4.9 & 15 & 1.27 & 2.57 & 1.39 \\
Range: & & $20-49$ & $18.5-23.5$ & $0.88-1.01$ & $3.0-5.3$ & & $0.98-1.70$ & $1.71-3.63$ & $1.11-1.65$ \\
\hline
\end{tabular}
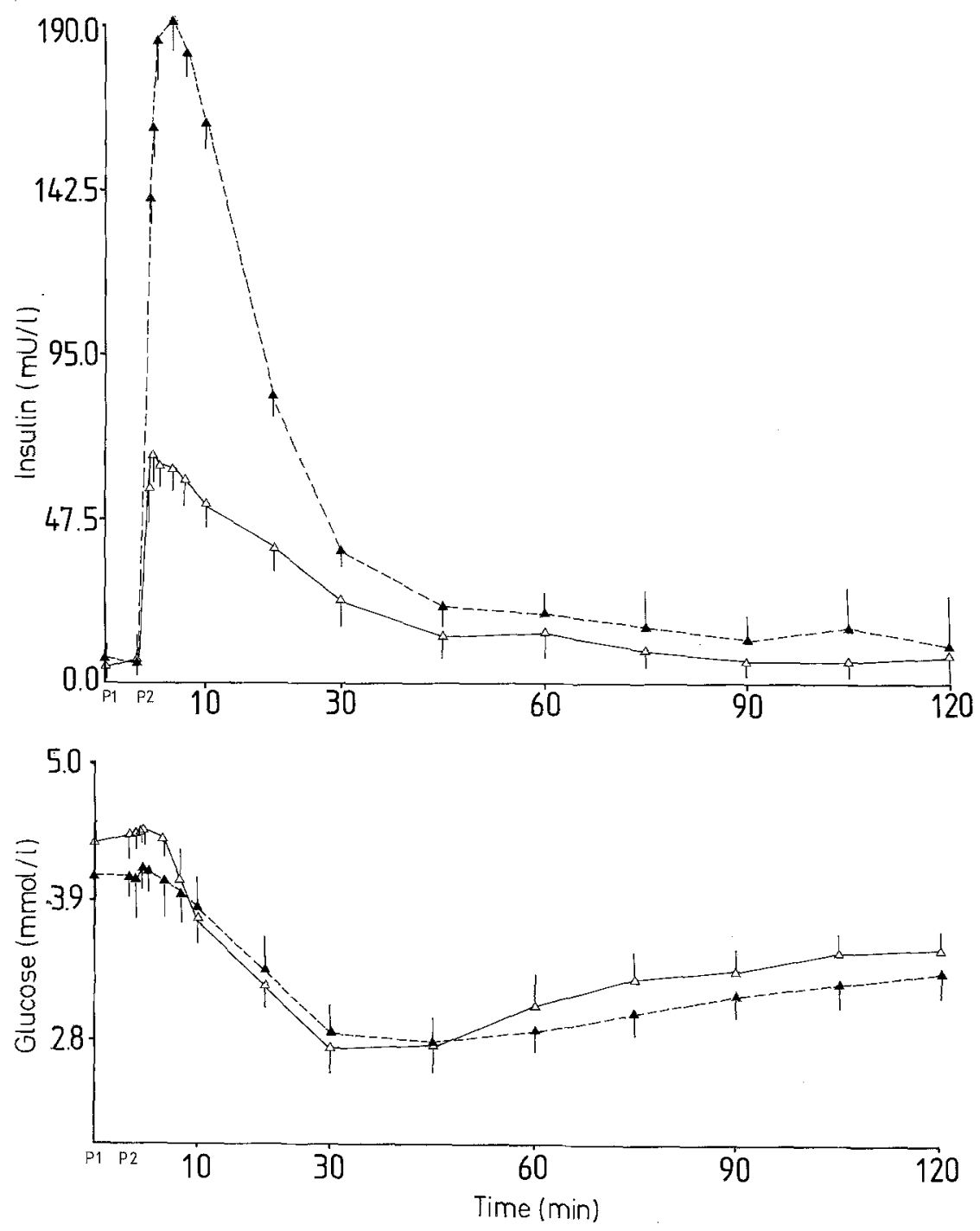

Fig. 1. Plasma insulin (upper) and glucose (lower) concentration profiles during a 120-min intravenous tolbutamide test (IVTT) in nine patients on (-- $\Delta---)$ or off $(-\Delta-)$ treatment with methandienone; data are shown as mean $\pm \mathrm{SE}$

\section{Statistical analysis}

The agreement between the estimates of $S_{I}$ derived from the IVGTT and IVTT procedures was tested using the Spearman rank correlation coefficient [24]. Deviations from a line of equality (i.e. along which $S_{I}$ (IVGTT] $=S_{I}$ (IVTT]) was quantified using a standardised residual mean square for each pair of values of $S_{l}$ computed. The deviation of the estimate made by the IVTT procedure was expressed as a residual $\left(d_{\mathrm{yx}}\right)$ of the line of equality, and plotted against the estimate made by the IVGTT procedure. Therefore:
$d_{\mathrm{yx}}=S_{I}[$ IVTT $]-S_{I}[$ IVGTT $]$.

Expressing the line as a linear equation in $\mathrm{x}$ of the general form:

$y=\mathrm{bx}+a$

the error residual mean square becomes:

$S_{\mathrm{yx}}=\left[\left(y_{1}-a-\mathrm{bx}_{i}\right)\right]^{2} /$ degrees of freedom (n of pairs) $=\left[\left(\mathrm{y}_{i}^{2}\left(a+\mathrm{bx}_{i}\right) y_{i}+\left(a+\mathrm{bx}_{i}\right)^{2}\right] / n\right.$. 
Table 2. Individual estimates of $S_{I}$ derived from the two procedures in Study B. Analyses listed according to status of treatment with methandienone. Legend otherwise as Table 1

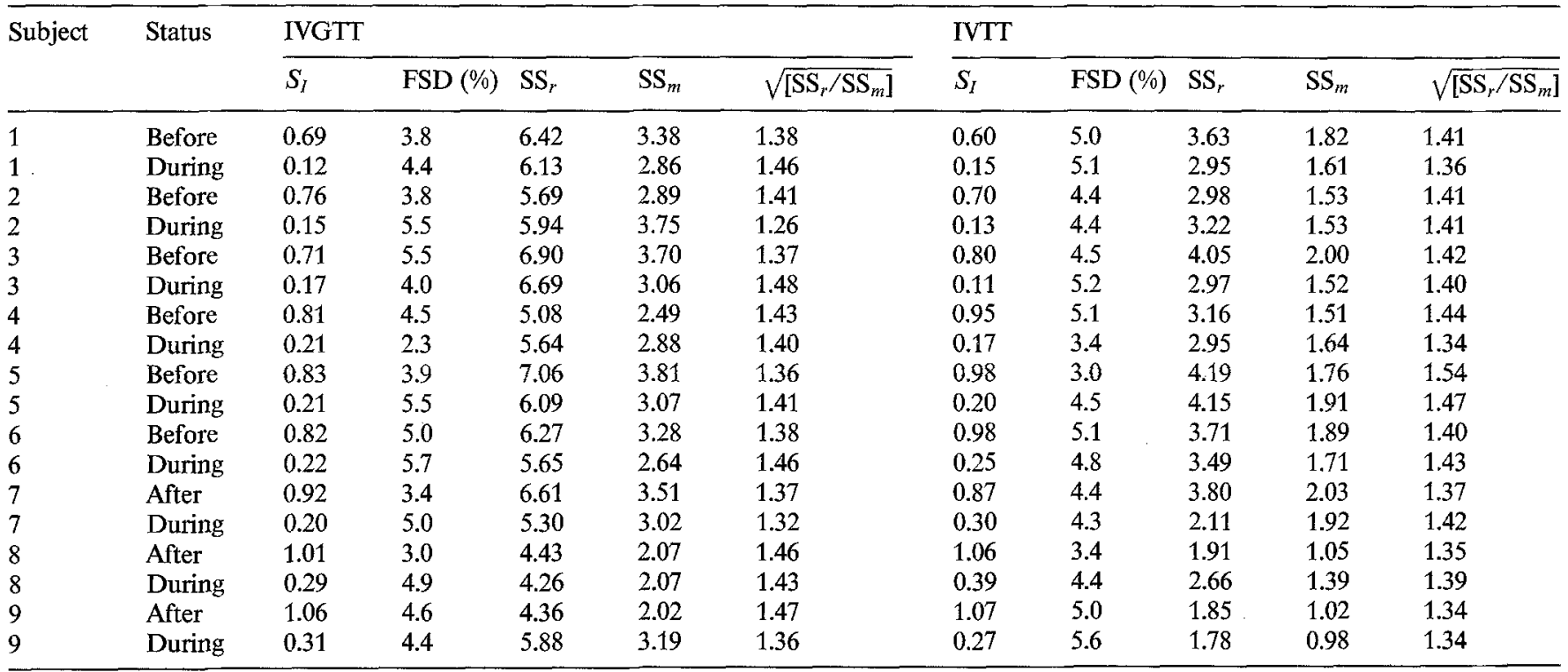

$S_{I}$ medians and ranges:

Before and after:

\section{IVGTT}

0.82

$[0.69-1.06]$

During:
0.21

[0.12-0.31]
IVTT

0.95

$[0.60-1.07]$

0.20

[0.11-0.39]
In this case, where $\mathrm{a}=0$ and $\mathrm{b}=1$, the expression reduces to:

$$
\begin{aligned}
S_{\mathrm{yx}} & =\left[\left(y_{i}-x_{i}\right)^{2} / n\right] \\
& =\left[d_{\mathrm{yx}} 2 / n\right] .
\end{aligned}
$$

The individual deviations were expressed as a percentage of the residual mean square and analysed using the Spearman rank correlation coefficient to test if the difference in the estimates from the two methods was associated with the magnitude of the original IVGTTderived estimate. The individual values of the differences, $d_{\mathrm{yx}}$, were tested for deviation from a Gaussian distribution with a Kolmogorov-Smirnov goodness-of-fit test $[23,24]$, and the proximity of the differences to a mean value of zero was estimated using a Wilcoxon paired-signed ranks test $[23,24]$.

Mann-Whitney U-tests and Wilcoxon paired signed-rank tests were used for all between-, and within-group parameter comparisons [24].

\section{Results}

\section{Study $A$}

Median age, BMI, $S_{1}$, FSD of the $S_{I}$ estimate, residual and measurement error sums of squares, and the suitability index for control subjects in Study A are summarised in Table 1. For the sexes combined, the total range of estimates of $S_{I}$ was contained within narrow bounds for both tests (IVGTT: $0.86-1.09 \mathrm{~min}^{-1} \mathrm{U}^{-1} 1$; IVTT: $\left.0.82-1.01 \mathrm{~min}^{-1} \mathrm{U}^{-1} 1\right)$, and the median values of $S_{I}$ also showed good agreement (IVGTT: $0.92 \mathrm{~min}^{-1} \mathrm{U}^{-1} 1$; IVTT: $0.89 \mathrm{~min}^{-1} \mathrm{U}^{-1} 1$; NS). The FSDs of the estimates ranged from 3 to $6 \%$. The suitability index showed no significant difference between the modelling analyses applied to the two methods and
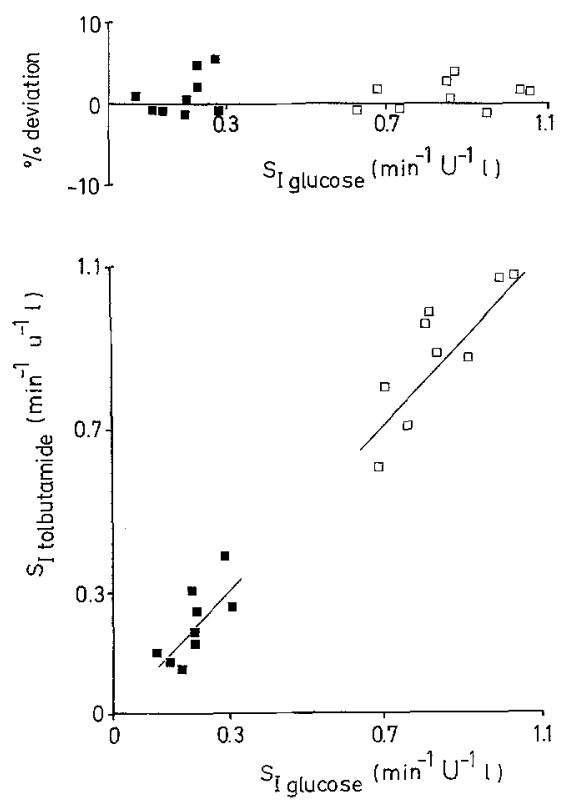

Fig. 2. Lower plots: Insulin sensitivity index, $S_{l}$, estimated from the results of (abcissa) intravenous glucose tolerance tests (IVGTTs) scattered against (ordinate) $S_{I}$ index estimates from intravenous tolbutamide tests (IVTTs) for patients off $(\square)$ or on ( $\square)$ treatment. Correlations were $r_{s}=0.71(p<0.05)$ off treatment and $0.69(p<0.05)$ on treatment $(n=9)$. Upper plots: Percentage deviations from the scatter plot described above, compared to the line of equality. The percentage deviation is the quantity $100\left[d_{\mathrm{yx}} / \mathrm{SR}_{\mathrm{yx}}\right]$ where $d_{\mathrm{yx}}$ is the individual absolute deviation for a given point, and $\mathrm{SR}_{\mathrm{yx}}$ is the residual sums of squares of the points from the line $y=b x$. The percentage deviation for the IVTT estimates of $S_{I}$ have been plotted against the corresponding values of $S_{I}$ determined from the IVGTT procedure; $r_{s}=0.36(n=9, \mathrm{NS})$ 
Table 3. Individual estimates of $S_{l}$ derived from the two procedures in Group C (miscellaneous subjects). Legend as in previous tables

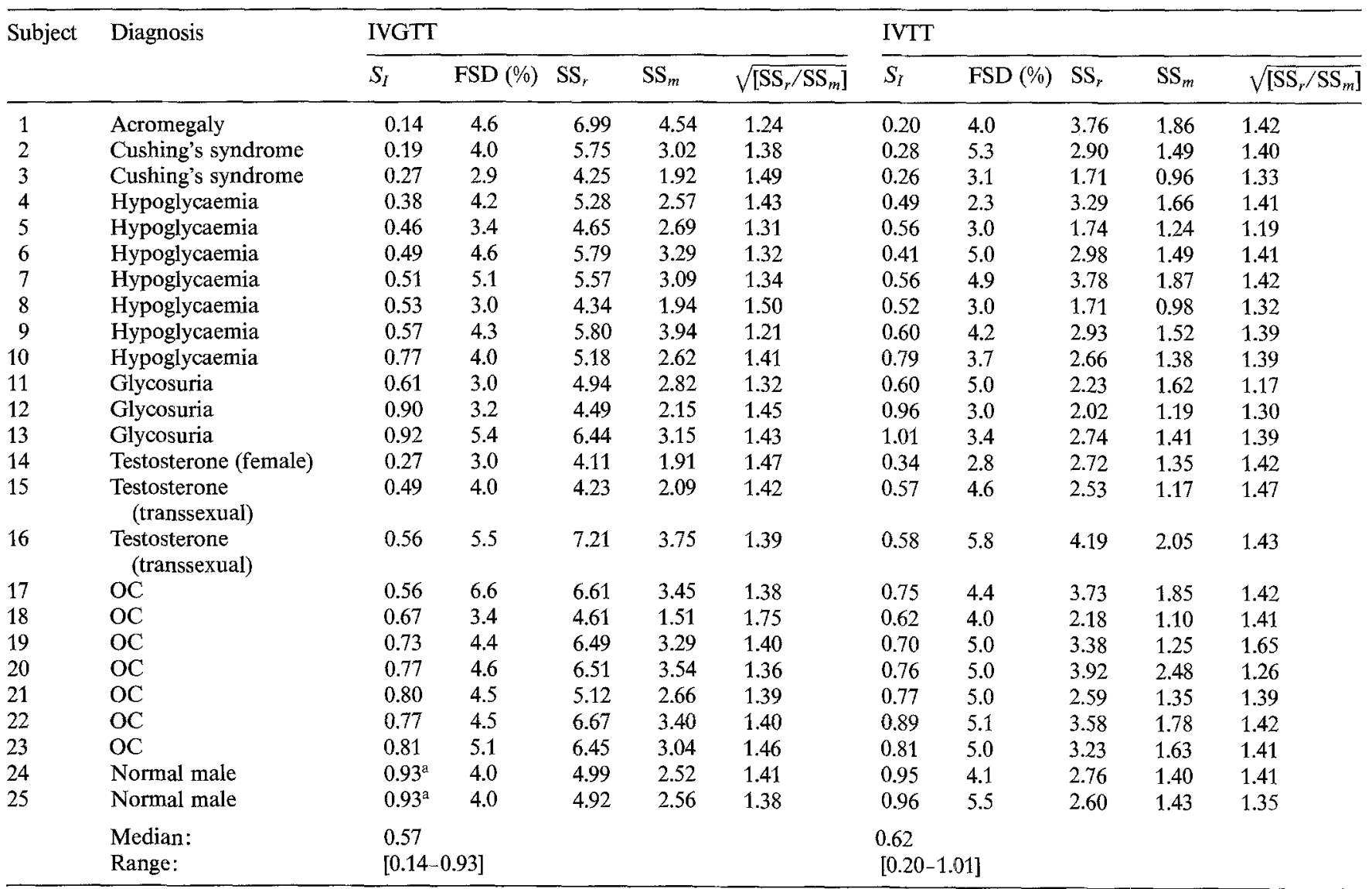

OC - oral contraceptive steroids. ${ }^{a}$ No recorded abnormalities or drugs

the ranges of estimates of this measure, for the whole study, were very consistent between the two methods (IVGTT: $1.09-1.78$, overall median 1.38; IVTT: $1.11-1.73$, overall median 1.42 ).

\section{Study $B$}

The IVGTT plasma glucose and insulin concentration profiles for the subjects of Study B have been described elsewhere and show marked impairment of glucose tolerance, associated with an elevated insulin response [8,9]. Mean IVTT plasma glucose and insulin concentration profiles for these subjects are given in Figure 1. The IVTT insulin response was markedly increased by treatment with methandienone $(p<0.001)$. A three-fold increase in both the peak insulin concentration and the area under the IVTT insulin profile was observed, although the time at which the insulin peak was reached was unchanged. The fasting plasma glucose levels were significantly reduced on treatment $(p<0.01)$, as previously observed. Although both the appearance of the nadir in the plasma glucose concentration and the recovery from hypoglycaemia were delayed $(p<0.01)$, the plasma glucose concentration at the nadir was unchanged. These results suggest that methandienone treatment causes insulin resistance.
Estimates of $S_{I}$ for the subjects of Study B are given in Table 2. Values of $S_{I}$ of the off treatment tests were comparable with the values for the Study A controls. However, treatment with methandienone resulted in a four-fold reduction in the index of insulin sensitivity, $S_{I}$, the same result that was obtained using the IVGTT. Individual IVTT data, and the residual differences between estimates from the two methods are plotted relative to the IVGTT-derived values of $S_{I}$ in Figure 2. The correlation between $S_{I}$ values derived from the two procedures was significant for both the on-, and off-treatment tests (off treatment $r_{s}=0.71$, $n=9, p<0.05$; on treatment $r_{s}=0.69, n=9, p<0.05$ ). No significant trend was found in the residual errors for either the off treatment or the on treatment analyses, and there was no evidence of a significant deviation from a zero mean value in these errors. The paired suitability indices for neither the off treatment nor the on treatment analyses showed any significant difference between the IVGTT and IVTT models. These observations suggest that the errors between the IVTT estimates of $S_{I}$ and those from the IVGTT were not influenced by systematic error, and were distributed normally along the line of equality. It is also evident from the analysis of the suitability indices that, as in the previous study, the numerical efficiencies of the 

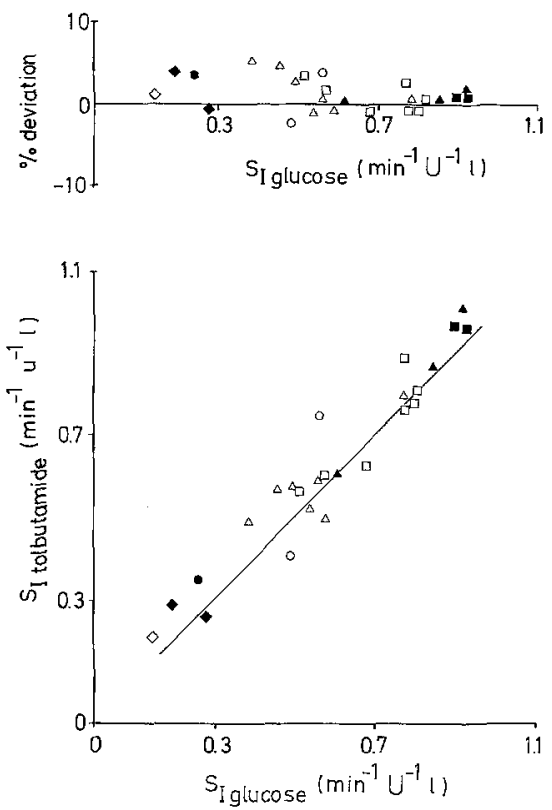

Fig.3. Lower plots: insulin sensitivity index, $S_{l}$, estimated from paired IVTTs and IVGTTs in patients with $(\diamond)$ acromegaly, $(\diamond)$ Cushing's syndrome, $(\Delta)$ hypoglycaemia, and $(\boldsymbol{\Delta})$ glycosuria, in $(O, O)$ transsexual subjects taking testosterone, in ( $\square$ ) women taking oral contraceptives, and in (ם) two normal men; $r_{s}=0.95$, $(n=25, p<0.001)$. Upper plots: scatter plot of percentage deviations from the line of equality as described in the legend to Figure 2; $r_{s}=0.21(n=25, \mathrm{NS})$

two models in producing acceptable Monte Carlo estimations of the observed data were not significantly different.

\section{Study C}

For the subjects in Study C, the correlation between $S_{I}$ values derived from the IVGTT and IVTT procedures was strong $\left(r_{s}=0.95, n=25, p<0.001\right)$. Individual estimates of $S_{I}$ are given in Table 3. The range of $S_{I}$ in Study $C$ was $0.20-1.01 \mathrm{~min}^{-1} \mathrm{U}^{-1} 1$ for the IVTTs and $0.14-0.93 \mathrm{~min}^{-1} \mathrm{U}^{-1} 1$ for the IVGTTs. Individual IVTT data, and the residual differences between estimates from the two methods are plotted relative to the IVGTT-derived values of $S_{I}$ in Figure 3. No significant trend was found in the residual errors and no deviation from a Gaussian distribution of zero mean was detectable. The suitability indices for the two methods were not significantly different and the ranges of this index for the two methods were very similar (IVGTT: 1.21-1.75, median 1.40; IVTT: $1.17-1.65$, median 1.41).

$S_{I}$ for the two normal males and two of the subjects with glycosuria gave values within the range encountered in the Study A control subjects. The seven women taking oral contraceptives, the two transsexuals receiving testosterone enanthate, one patient with glycosuria, and the cases under investigation for reactive hypoglycaemia all showed reduced insulin sensitivity compared with controls. The two patients with Cush- ing's syndrome, the patient with acromegaly and the female patient receiving testosterone enanthate showed the lowest values of $S_{I}$.

\section{Discussion}

Aspects of insulin insensitivity, particularly hyperinsulinaemia, are a common thread running through the associated conditions of obesity [25], Type 2 (non-insulin-dependent) diabetes mellitus [26], aging [27], hypertension [28], and cardiovascular disease [29, 30]. Insulin resistance also results from glucocorticoid excess, and from the administration of a variety of gonadal steroids [31] and is a feature of certain pathological conditions, such as acanthosis nigricans [32] and polycystic ovary syndrome [33]. Evaluation of the importance of insulin insensitivity in all these conditions has been limited by the nature of the techniques available for its quantification.

The reference technique for measurement of insulin sensitivity is generally accepted to be the euglycaemic clamp, according to which the rate of glucose disposal at a given concentration of insulin is determined in a steady state. However, in order to overcome the mutual feedback by which glucose and insulin concentrations are maintained, continuous infusions of insulin and glucose are required and there is sustained hyperinsulinaemia throughout the procedure. Glucose clamp techniques are thus invasive research procedures that impose a physiological equilibrium on the experimental subject that is far from normal [13].

An alternative to such steady-state techniques is mathematical modelling of dynamic changes in plasma glucose and insulin concentrations following a disturbance in either of these parameters. One of the most promising of these techniques is the application of the minimal model of glucose dynamics to predict plasma glucose concentrations from plasma insulin concentrations following intravenous injection of glucose. The parameter of insulin sensitivity thus derived is termed $S_{I}$ [1]. By comparison with the euglycaemic clamp, the IVGTT is relatively easy to perform and may be considered as a diagnostic procedure. However, it requires injection of a large volume of substance, with a small but significant risk of thrombophlebitis ensuing. In the present study we have explored the application of the minimal model of glucose dynamics to changes in plasma glucose and insulin concentrations following stimulation of insulin secretion by intravenous injection of tolbutamide. According to this procedure a smaller volume of substance is injected and the resulting fall in plasma glucose concentrations is transient and rarely sufficient to cause symptoms of hypoglycaemia. Furthermore, it was of interest to explore the comparability of values of $S_{I}$ derived conditions of both hypo-, and hyperglycaemia. 
It is apparent from the results presented above that estimates of $S_{I}$ derived from the IVGTT and IVTT are virtually identical and that this identity holds over a wide range of values of $S_{I}\left[0.11-1.07 \mathrm{~min}^{-1} \mathrm{U}^{-1} 1\right]$. The assumptions made in order to adapt the model to the plasma glucose and insulin concentration dynamics associated with the IVTT thus appear to hold, and support is given for the generality of the minimal model in conditions when dynamic changes in plasma glucose concentrations result from an increase in insulin secretion.

The subjects of Study A are probably the largest group of healthy, normal individuals yet assessed by minimal modelling techniques. The mean values of $S_{I}$ thus obtained compare well with those reported for similar individuals by Chen and co-workers [34], although the mean $S_{I}$ for the small group they describe $\left[n=10, S_{I}=0.65 \mathrm{~min}^{-1} \mathrm{U}^{-1}\right.$ l] is lower than we found, perhaps due to the predominance of subjects with body weights greater than $100 \%$ of ideal. Preliminary studies we have undertaken indicate that $S_{I}$ is highly sensitive to the degree of obesity, and this may account for the narrow range of values of $S_{I}$ we find in our group of carefully selected normal subjects.

The euglycaemic clamp remains the reference method for measurement of insulin sensitivity, and it is necessary to consider the validity of minimal modelbased estimates of insulin sensitivity in relation to comparable clamp-based estimates. This has been examined in detail by Beard and co-workers [35], who compared clamp-derived estimates of $S_{I}$ with estimates derived from standard IVGTTs and a modified IVGTT protocol in which a bolus injection of tolbutamide was given $20 \mathrm{~min}$ after the injection of glucose. This modification was introduced to maximise the insulin concentrations associated with the declining plasma glucose concentration during the IVGTT, and thus minimise the FSD of the estimates of $S_{I}$ thus obtained. It also separated the phase of the IVGTT during which redistribution of the glucose load throughout the body is occurring from the phase in which plasma glucose concentration falls, and insulin-dependent glucose decay occurs. No correlation was found between the clamp- and standard IVGTT-derived estimates of $S_{b}$ whereas a strong correlation was obtained between the clamp- and modified IVGTT-derived estimates. This appeared to be due to the considerably lower FSDs of the estimates of $S_{I}$ for the modified test compared with the standard test [means: $3.0 \%$ vs $17.4 \%$ respectively]. In the present study the IVGTTs performed did not included the tolbutamide modification. However, the dose of glucose we used was $0.5 \mathrm{~g}$ per $\mathrm{kg}$ body weight compared with the $0.3 \mathrm{~g}$ per $\mathrm{kg}$ dose used by Beard et al. [35] in their standard IVGTT. Also, we have zeroweighted results during the first $3 \mathrm{~min}$ of the test. Our protocol thus results in an augmented insulin response compared with the standard IVGTT of Beard et al., [35] and also minimises the effect of redistribution of the glucose dose. The mean FSD of the estimates of $S_{I}$ we obtain for both the IVGTT and IVTT in the present study is $4.4 \%$, which compares well with that reported for the tolbutamide-modified test. Our results may therefore be expected to correlate well with those obtained with the modified IVGTT and, by implication, with the euglycaemic clamp.

Support for the validity of the estimates of $S_{I}$ reported here is provided by the discrimination that is apparent between the estimates of $S_{I}$ for the healthy normal subjects in Study A, and the estimates in patients in whom diminished insulin sensitivity has previously been demonstrated or would be expected. With regard to Study B, previous studies of the influence of methandienone on carbohydrate metabolism showed that the insulin response to oral or intravenous glucose was dramatically increased to an extent greater than that of any other steroid we have studied $[8,9]$, an observation consistent with reduced sensitivity to insulin. In Study C, subjects with acromegaly and Cushing's syndrome and patients undergoing other steroid treatments were studied. These conditions and treatments are each known to be associated with insulin resistance $[29,36,37]$ and estimates of $S_{I}$ were consistent with this in all cases.

Minimal modelling of both the IVGTT and IVTT would appear to give a valid measure of insulin sensitivity. The extent to which these methods are used will depend on future experience gained both in research and clinical practice. The value of measuring $S_{I}$ has not yet been established, primarly because of the lack of a suitable method with which to investigate insulin sensitivity in significant numbers of cases. In its present form, the minimal model methodology requires that the subject under investigation has a sufficient pancreatic insulin response and this would appear to be a limitation where overt diabetes is under investigation. However, it may be that in those cases in which insulin resistance is associated with an augmented insulin response and hyperinsulinaemia, that measurement of $S_{I}$ will prove to be of greatest value. The longterm consequences of elevated plasma insulin concentrations are only now becoming apparent in relation to coronary heart disease risk $[38,39]$. Whether diminished insulin sensitivity predisposes to risk of future morbidity will only be determined if straightforward, effective methods for its measurement are established.

Acknowledgements. The results reported here were obtained with the clinical and technical assistance of the staffs of the St. Mary's Hospital Medical School Metabolic Unit Day Ward and Laboratory; their assistance is gratefully acknowledged. Financial support for this study was provided by the National Institutes of Health, USA, contract no. NICHD-CE-84-1.

\section{References}

1. Bergman RN, Phillips LS, Cobelli C (1981) Physiological evaluation of factors controlling glucose tolerance in man: measurement of insulin sensitivity and beta-cell pancreatic responsivity 
from the response to intravenous glucose. J Clin Invest 68: 1456-1467

2. DeFeo P, Perriello G, DeCosmo S, Ventura MM, Campbell PJ, Brunetti P, Gerich JE, Bolli GB (1986) Comparison of glucose counterregulation during short-term and prolonged hypoglycaemia in normal humans. Diabetes 35: 563-569

3. Garber AJ, Cryer PE, Santiago JV, Haymond MW, Pagliara AS, Kipnis DM (1976) The role of adrenergic mechanisms in the substrate and hormonal response to insulin-induced hypoglycaemia in man. $\mathrm{J}$ Clin Invest 58: 7-15

4. Gerich JE, Schneider V, Dippe SE, Langlois M, Noacco C, Karam JH, Forsham PH (1974) Characterisation of glucagon responses to hypoglycaemia in man. J Clin Endocrinol Metab 38: $77-82$

5. Feldman J, Plonk J, Bivens C (1976) The role of cortisol and growth hormone in the counterregulation of insulin-induced hypoglycaemia. Horm Metab Res 25: 227-232

6. Lucas KJ, Karounos DG, Ellis GJ, Morris MA, Pisetsky DS, Feinglos MN (1986) The intravenous insulin tolerance test in Type I diabetes. Res Comm Chem Pathol Pharmacol 53: 331-345

7. Unger RH, Orci L (1976) Physiology and pathophysiology of glucagon. Physiol Rev 56: 778-826

8. Godsland I, Shennan NM, Wynn V (1986) Insulin action and dynamics modelled in patients taking the anabolic steroid, Dianabol. Clin Sci 71: 665-673

9. Landon J, Wynn V, Houghton BJ, Cooke JNC (1962) Effects of the anabolic steroid, methandienone, on carbohydrate metabolism in man. Metabolism 11: 501-511

10. Cramp DG (1967) New automated method for measuring glucose by glucose oxidase. J Chem Pathol 20: 910-912

11. Albano JDM, Ekins RP, Maritz G, Turner RC (1972) A sensitive, precise radioimmunoassay of serum insulin relying on charcoal separation of bound and free hormone moieties. Acta Endocrinol 70: 487-509

12. Bergman R, Beard JC, Chen M (1986) The minimal modelling method. Assessment of insulin sensitivity and beta-cell function in vivo. In: Clarke W, Warner J, Pohl SJ (eds) Methods in diabetes research, Vol II: Clinical methods. John Wiley, New York, pp 15-34

13. Bergman R, Finegood DT, Ader M (1985) Assessment of insulin sensitivity in vivo. Endocr Rev 6: 45-86

14. Rizza RA, Mandarino LJ, Gerich JE (1981) Dose-response characteristics for effects of insulin on production and utilization of glucose in man. Am J Physiol 240: E630-E639

15. Bergman RN, Bucolo RJ (1974) Interaction of insulin and glucose in the control of hepatic glucose balance. Am J Physiol 227: $1314-1322$

16. Best, JD, Taborsky Jr, GJ, Halter JB, Porte Jr D (1981) Glucose disposal is not proportional to plasma glucose level in man. Diabetes 30: $847-850$

17. Marquardt DW (1963) An algorithm for least squares estimation of nonlinear parameters. SIAM J Soc Ind Appl Math 2: 431-441

18. DiStefano III JJ (1982) Algorithm, software and sequential optimal sampling schedule design for pharmacokinetic and physiologic experiments. Math Comput Simul 24: 531-534

19. Leigh JR (1983) Essentials of nonlinear control theory. Peter Peregrinus, London

20. Carson ER, Cobelli C, Finkelstein L (1983) The mathematical modeling of metabolic and endocrine systems. John Wiley, New York

21. Bergman RN, Ider YZ, Bowden CR, Cobelli C (1979) Quantitative estimation of insulin sensitivity. Am $J$ Physiol 236: E667-E677
22. Finkelstein L, Carson ER (1985) Mathematical modelling of dynamic biological systems. Research Studies Press, John Wiley, New York

23. Morrison NK (1982) SPSS-11: The SPSS batch system for the DEC PDP-11. McGraw Hill, New York

24. Siegel S (1956) Nonparametric statistics for the behavioral sciences. McGraw Hill/Kogakusha, New York

25. DeFronzo RA (1982) Insulin secretion, insulin resistance and obesity. Int J Obes 6 [Suppl]: 73-82

26. Reaven GM (1983) Insulin resistance in noninsulin-dependent diabetes mellitus: does it exist and can it be measured? Am J Med 74: 3-17

27. Rowe J, Minaker KL, Pallotta JA, Flier JS (1983) Characterisation of the insulin resistance of aging. $J$ Clin Invest 71: 1581-1587

28. Christlieb AR, Krolewski AS, Warram JH, Soeldner JS (1985) Is insulin the link between hypertension and obesity? Hypertension (II) $7: 54-57$

29. Stout RW (1979) Diabetes and atherosclerosis - the role of insulin. Diabetologia 16: 141-150

30. Stout RW (1977) The relationship of abnormal circulating insulin levels to atherosclerosis. Atherosclerosis 27:1-13

31. Wynn V, Adams PW, Godsland I, Melrose J, Nithyananthan R, Oakley NW, Seed M (1979) Comparison of effects of different combined oral contraceptive formuations on carbohydrate and lipid metabolism. Lancet 1979 (1): 1045-1049

32. Stuart CA, Peters EJ, Prince MJ, Richards G, Cavallo A, Meyer WJ (1986) Insulin resistance with acanthosis nigricans: the role of obesity and androgen excess. Metabolism 35: 197-205

33. Burgher GA, Givens JR, Kitabchi AE (1980) Correlation of hyperandrogenism with hyperinsulinism in polycystic ovarian disease. J Clin Endocrinol Metab 50: 113-116

34. Chen M, Bergman RN, Pacini G, Porte Jr D (1985) Pathogenesis of age-related glucose intolerance in Man. Insulin resistance and decreases beta-cell function. J Clin Endocrinol Metab 60: 13-20

35. Beard JC, Bergman RN, Ward WK, Porte Jr D (1986) The insulin sensitivity index in nondiabetic man: correlation between clampderived and IVGTT-derived values. Diabetes 35: 362-369

36. Hansen I, Tsalikian E, Beaufrere B, Gerich J, Haymond M, Rizza $\mathrm{R}$ (1986) Insulin resistance in acromegaly: defects in both hepatic and extrahepatic insulin action. Am J Physiol 250: E269-E273

37. Karnieli E, Cohen P, Barzilai N, Ish-Shalom Z, Armon M, Rafaelov R, Barzilai D (1985) Insulin resistance in Cushing's syndrome. Horm Metab Res 17: 518-521

38. Pyorala K, Savolainen E, Lehtovirta E, Punsar $S$, Siltanen $P$ (1979) Glucose tolerance and coronary heart disease: Helsinki Policemen Study. J Chron Dis 32: 729-745

39. Ducimetiere $\mathbf{P}$, Eschwege E, Richard J, Claude J, Elgrishi I (1979) Relationship of glucose tolerance to prevalence of ECG abnormalities and to annual mortality from cardiovascular disease: results of the Paris Prospective Study. J Chron Dis 32: $759-766$

Received: 4 August 1986

and in revised form: 27 March 1987

Dr. N. M.Shennan

The Cavendish Clinic

21 Wellington Road

St. John's Wood

London NW8 9SQ

UK 